\title{
Intensity Modulation of Pseudocolor Images
}

BioTechniques 25:124-128 (July 1998)

\author{
Lee E. Hinman and Paul J. Sammak \\ University of Minnesota, Minneapolis, MN, USA
}

\begin{abstract}
Fura-2 is a popular real-time fluorescent indicator used to image intracellular, free calcium concentrations $\left(\left[\mathrm{Ca}^{2+}\right]_{i}\right)$ in living cells. One difficulty with fura-2-based digital imaging is how to best display the $\left[\mathrm{Ca}^{2+}\right]_{i}$ information that is acquired. Typically, only the $\left[\mathrm{Ca}^{2+}\right]_{i}$ as pseudocolor data are displayed, and the localization and intensity of the dye are not presented. This paper describes a technique that allows for the re-introduction of the intensity information. Recovery of the intensity information would de-emphasize regions with a poor signal-to-noise ratio and would more accurately report variations of sample morphology. A second advantage of this technique is that it requires no additional hardware since it is completely software based and can be run on all the major operating systems.
\end{abstract}

\section{INTRODUCTION}

In the eleven years since its invention (3), fura-2 has been described in many publications for its use as an indicator for intracellular, free calcium concentrations $\left(\left[\mathrm{Ca}^{2+}\right]_{\mathrm{i}}\right)$. The amazing popularity of the dye has been due to its independence from dye concentration, path length, uneven illumination and absolute sensitivity of the instrument and its ease of loading and resistance to photobleaching. The first four properties are due to the fact that the concentration of $\left[\mathrm{Ca}^{2+}\right]_{\mathrm{i}}$ is proportional to the ratio of the fluorescence of the dye at two excitation wavelengths (340 and $385 \mathrm{~nm}$ ). Taking the ratio of the fluorescent images at two different wavelengths removes the contribution of dye concentration, path length, uneven illumination and absolute sensitivity of the instrument because both wavelengths would be effected the same way, and the result of the division cancels these affects. Fluorescence ratio imaging solves many problems; however, it introduces several new problems, including how to accurately display all of the data that were acquired.
To make it easier for the human eye to detect small changes in value, the image can be pseudocolored. The human eye can distinguish among hundreds of colors but only 30 gray levels (6). It is also easier for the human eye to accurately identify that two pixels separated by a large distance are both red, whereas it is difficult to identify that the same two pixels have the same gray value. In this way, pseudocoloring makes it easier for the human eye to detect the subtle changes in ratios or $\left[\mathrm{Ca}^{2+}\right]_{i}$. However, this does nothing for the other problems of displaying the ratio image.

One problem is that, in the ratio picture, the original intensity information has been lost. This is best illustrated by the fact that the same ratio information can come from very different intensities. If a ratio pixel had the same intensity value in the 340 and 385 picture, the ratio would be the same since $1 / 1=1$ and $255 / 255=1$. Both ratio pixels would record the same ratio value, but the initial data are quite different. This intensity information is very useful in determining where data are likely to be reliable (high signal to noise) and where data are likely to be unreliable (low signal to noise). Another problem is the loss of information about dye localization.

These problems were previously noticed by Tsien and Harootunian (7), and a solution was proposed. Changes in color were mapped to $\left[\mathrm{Ca}^{2+}\right]_{\mathrm{i}}$, and the intensity of the color was mapped to the intensity of the original signal. In this way, all the data were retained in the final display. Unfortunately, the method relied heavily on dedicated hardware and software. This limited the widespread implementation of this method.

In 1992, Gustafson and Magnusson (4) proposed another solution. The analog 340-nm signal was connected to the Red video input of an red green blue (RGB) monitor, and the 380$\mathrm{nm}$ signal was connected to the green video input. The ratio of the 340/380 signal was represented as the ratio of red/green, which would appear as a shade of yellow. The major drawback of this technique was that it still relied on specific hardware.

Our paper outlines a general method that will achieve results similar to those of Tsien and Harootunian. In addition, this method does not rely on any specific hardware but is instead done completely with image-processing routines, which can be performed using commercially available software. While this method can be used to simply improve the final 
display of fura- 2 data, it can also be incorporated into the image analysis to achieve greater accuracy with fura- 2 measurements.

\section{MATERIALS AND METHODS}

\section{Computer Hardware and Software}

The procedure for intensity modulating $\left[\mathrm{Ca}^{2+}\right]_{\mathrm{i}}$ pseudocolor images was as follows. The $\left[\mathrm{Ca}^{2+}\right]_{i}$ data were mapped to the hues of the colors in the final image, and the $\left[\mathrm{Ca}^{2+}\right]_{\mathrm{i}}$ independent fluorescence intensity was mapped to the intensity of the colors in the final display. The $\left[\mathrm{Ca}^{2+}\right]_{\mathrm{i}}$ data were derived from the ratio of fluorescent intensity of images taken at 340 and $385 \mathrm{~nm}$ excitation. The intensity data were derived from the isobestic sum of the two images (see below).

Intensity modulation was designed using IPLab Spectrum $^{\mathrm{TM}}$ (Signal Analytics, Vienna, VA, USA) on a Power Macintosh ${ }^{\circledR}$ (Apple Computer, Cupertino, CA, USA). However, Adobe ${ }^{\circledR}$ Photoshop ${ }^{\circledR}$ (Adobe Systems, Mountain View, CA, USA) with the Image Processing Toolkit (Reindeer Games, Asheville, NC, USA) will be used as a specific example because of Photoshops large installed base and the Image Processing Toolkits cross-platform capabilities. Note that neither program is specifically required to perform this method. The image-processing routines that are required are: $(i)$ image arithemetic (addition and division), (ii) custom look up tables (LUT) and (iii) conversion between RGB and hue saturation value (HSV) color space. The first two are included in almost every image-processing program, the conversion between color spaces is less common. If your image-processing program does not support HSV, a suitable algorithm for converting to and from HSV space (written in the $\mathrm{C}$ programming language) can be found in Reference 1 .

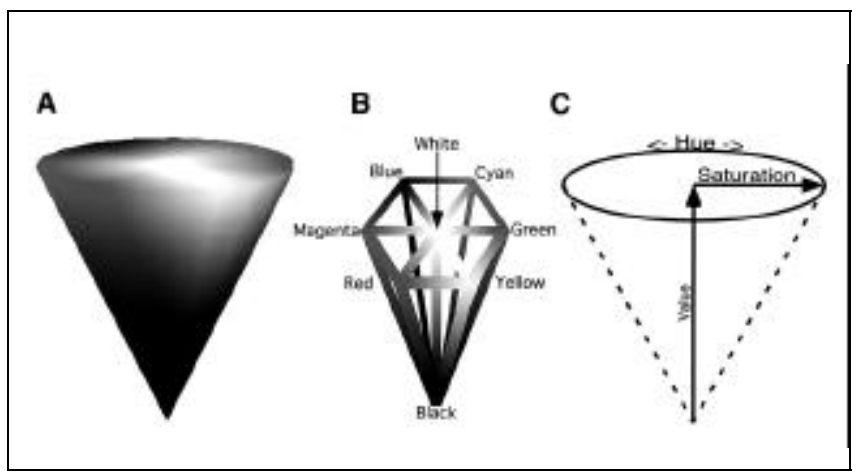

Figure 1. (A) A filled three-dimensional (3-D) cone representing the HSV color space. Note that the colors converge to white at the center of the cone and that the colors converge on black at the tip of the cone. (B) A hexagonal cone representing HSV color space. Note here the placement of the colors and that they are all separated by $60^{\circ}$. Also note that white and black are unique points defined by a value of $100 \%$ and a saturation of $0 \%$ and a value of $0 \%$, respectively. (C) A line drawing of a cone representing HSV color space. Note here that hue is measured radially in degrees. Red is usually assigned $0^{\circ}$. Saturation increases as you move perpendicularly away from the axis of the cone and is expressed as a percent. Value increases as you move from the tip of the cone up the axis and is also measured in percent.

\section{Ratio and Isobestic Images}

Most commercial software designed for $\left[\mathrm{Ca}^{2+}\right]_{\mathrm{i}}$ imaging will ratio and calibrate the images for you, in which case you should use the manufacturer's routines. If an independent software program is being designed, necessary formulas and considerations can be found elsewhere $(3,7)$.

One of the unique properties of fura- 2 is that the emitted intensity at the excitation wavelength of $360 \mathrm{~nm}$ is insensitive to the changes in $\left[\mathrm{Ca}^{2+}\right]_{\mathrm{i}}(3)$. The fluorescence intensity at this wavelength is mostly due to the amount of dye loaded or, more fundamentally, the strength of the signal and sample thickness. A 360-nm picture can be acquired directly, but most researchers find this undesirable since it requires additional filters, exposure time and extra storage space. A popular alternative is combining the 340 and $385 \mathrm{~nm}$ wavelength pictures arithemetically such that the result is insensitive to the concentration of $\left[\mathrm{Ca}^{2+}\right]_{\mathrm{i}}(2)$. This method saves a great deal of time during acquisition and also saves storage space, since the isobestic image can be calculated whenever it is needed. Either the calculated or measured isobestic image will work with this method.

\section{Look Up Table}

To increase the ability of the human eye to distinguish the changes in $\left[\mathrm{Ca}^{2+}\right]_{i}$, the $\left[\mathrm{Ca}^{2+}\right]_{\mathrm{i}}$ data are mapped to a color in the final image. This is achieved by creating an LUT that it specifies which $\left[\mathrm{Ca}^{2+}\right]_{\mathrm{i}}$ data get mapped to which color (e.g., $20 \mathrm{nM}=$ blue, and $200 \mathrm{nM}=$ red). The choice of the correct LUT is the most crucial part of this process. In this method, $\left[\mathrm{Ca}^{2+}\right]_{i}$ is mapped to hue. A LUT should be chosen such that only varies in hue and does not vary in saturation or value. The best way to explain which colors only contain hue is in the HSV color space.

Figure 1 shows the HSV color space in three equivalent representations. HSV can be represented as either a cone or cylinder. We have chosen to represent HSV as a cone because we feel this correlates best to the data acquired in fura- 2 digital imaging. In this representation, hue is the angle (in degrees) perpendicular to the major axis of the cone (Figure 1C). Saturation is the radius of the cone and is measured in percent, increasing from 0 at the center of the cone to 100 at the outer rim (Figure 1C). Value is the height of the cone and is also measured in percent, increasing along the axis of the cone with 0 at the tip of the cone and 100 at the top (Figure 1C).

While this explains how the variables are represented, it does not explain what they mean. Hue is the color as described by its characteristic wavelength. This is how most artists think of colors, as groupings of blues, reds, yellows etc. Saturation involves the amount of color that is present. As saturation decreases, the color gets closer to white. Value involves the amount of black in the color, as demonstrated by traveling up the axis of the cone where hue and saturation are zero. The axis of the cone is actually a gray-scale gradient, demonstrating that as value increases, the color contains less and less black (Figure 1B).

This method requires mapping $\left[\mathrm{Ca}^{2+}\right]_{\mathrm{i}}$ to just hue. These are the colors on the outer rim of the cone (Figure 1). We 
recommend a LUT that progresses from cyan $\rightarrow$ green $\rightarrow$ yellow $\rightarrow$ red $\rightarrow$ magenta, as blue often creates problems in reproduction (see Discussion). This LUT follows the social norms of blue-green being resting or cool and reds and magenta being hot or active. This LUT also follows a spectral progression that is often easier for human eye to distinguish as a gradient and easier for software to generate. And lastly, this LUT is the one most commonly used for $\left[\mathrm{Ca}^{2+}\right]_{i}$ pictures.

In Photoshop, this is achieved by first changing the mode of the image from gray scale to Index Color. This is necessary so that a custom LUT or color table can be chosen. Next, the new LUT is defined using the Color Table command. The LUT we recommend must be entered manually using the Custom command; however, it can be saved and reloaded for subsequent pictures.

\section{RGB $\rightarrow$ HSV Conversion}

Applying the LUT to the gray-scale image results in RGB pseudocolor image. This image in now converted to HSV space, which will produce three images: hue, saturation and value.

In Photoshop, this is done by first changing the mode of the image from Index Color to RGB. This is necessary since most conversions to and from HSV space cannot take input from the multiple LUTs of Index Color; the RGB LUT on the other hand is constant. To convert from RGB to HSV space, we used the Color Space Plugin in the Image Processing Toolkit. Simply choose the $R G B \rightarrow H S V$ option, and the plugin converts the image for you. This results in a single image with three channels. The names of the channels are slightly misleading due to a limitation in Photoshop; the red channel is hue, the green channel is saturation and the blue channel is value. Running the Split Channel command results in three separate gray-scale images of the corresponding HSV triplet.

\section{HSV $\rightarrow$ RGB Conversion}

At this point, convert the image from HSV back to RGB space, with a critical substitution. Instead of using the initial Value picture, substitute the Isobestic picture. In other words, select the hue and saturation image from the RGB $\rightarrow \mathrm{HSV}$ split and the isobestic picture as the value image. Now convert these three images to RGB.

In Photoshop, this is done by first merging the three grayscale images into a single image. First run the Merge Channels command. Even though we are merging an HSV image, choose RGB and 3 channels; this is because Photoshop does not have built-in HSV support. Remember that the red channels is actually hue, the green is saturation and the blue is value. Now run the Color Space Plugin from the Image Processing Toolkit, and choose the $H S V \rightarrow R G B$ option. This results in a single image.

The resulting picture should only show color where there was a significant signal in the originally acquired data. Furthermore, the intensity of the color is now proportional to the intensity of the $\left[\mathrm{Ca}^{2+}\right]_{i}$-independent signal. This results in a visual weighting of the data based on the original signal-tonoise ratio. Areas that had large amounts of fura-2 are bright, and areas that had little fura- 2 are dim and are not noticed by the human eye.

\section{RESULTS}

Primary rat alveolar Type II cells were prepared as previously described (5). Confluent cell monolayers were loaded with fura- 2 and were wounded by scratching with a needle while ratiometric images were recorded with a cooled chargecoupled device (CCD) camera (Photometrics, Tucson, AZ, USA). Additional details can be found elsewhere (5).

Figure 2, A and B, shows pictures of the response of fura-2 after excitation with 340 and $385 \mathrm{~nm}$ light, respectively, in alveolar Type II cells $35 \mathrm{~s}$ after scratch wounding. In Panel B, near the wound edge, it is possible to see that the fluorescence intensity is less than it is far away from the wound. This is because fura- 2 fluoresces less strongly at $385 \mathrm{~nm}$ when $\left[\mathrm{Ca}^{2+}\right]_{\mathrm{i}}$ is increased. Panel A is the matched $340 \mathrm{~nm}$ picture, yet it is almost impossible to detect that fura- 2 intensity at $340 \mathrm{~nm}$ has increased because of the increase in $\left[\mathrm{Ca}^{2+}\right]_{i}$.

Figure 2C shows the weighted average of Panels A and B. It is easy to see that all cells are of similar intensity, even the ones experiencing a rise in $\left[\mathrm{Ca}^{2+}\right]_{\mathrm{i}}$. This is because this image is insensitive to the changes in $\left[\mathrm{Ca}^{2+}\right]_{\mathrm{i}}$. While this image gives no data on the changes in $\left[\mathrm{Ca}^{2+}\right]_{\mathrm{i}}$, it does provide important information. It shows where the signal was strongest and where it was not even present. This image also represents the signal-to-noise ratio for each individual pixel, since statistical photon noise dominates other intensity-independent sources of noise in these pictures.

When the ratio of these two pictures is taken, as in Figure $2 \mathrm{D}$, it is very easy to see that $\left[\mathrm{Ca}^{2+}\right]_{\mathrm{i}}$ was elevated near the wound edge. But making good comparisons between areas is very difficult, due to the small changes in gray value. It is also important to notice the insertion of errors in the display. In the

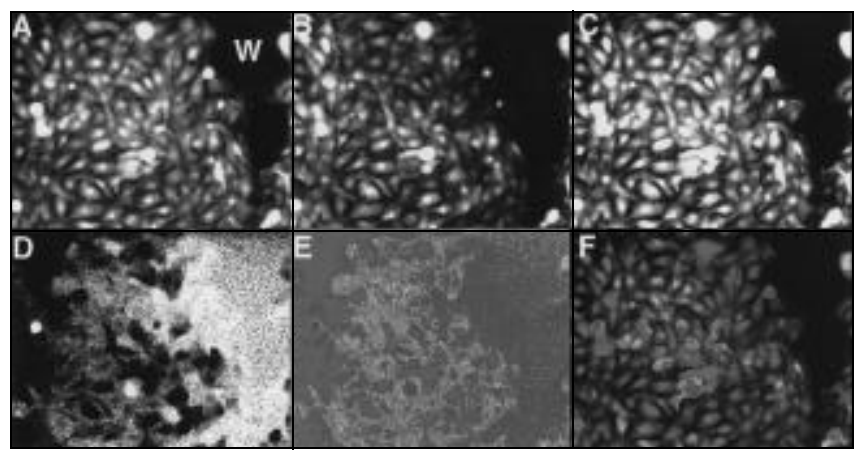

Figure 2. (A) A 340-nm excitation fluorescent picture of Alveolar Type II cells $35 \mathrm{~s}$ after wounding. (B) The same cells, excited with 385-nm light. (C) An isobestic picture of the cells. The picture was obtained by taking the weighted average of Panels A and B, specifically $0.8 \times 1340+0.2 \times 1385=$ Isobestic. The weights were determined empirically. (D) The ratio of Panels $A: B$, which was then calibrated to $\left[\mathrm{Ca}^{2+}\right]_{i}$. (E) The same information as Panel $\mathrm{D}$ except the gray values have been pseudocolored. It is easy to see the report of $\left[\mathrm{Ca}^{2+}\right]_{\mathrm{i}}$ in the wound area is an artifact. $(\mathrm{F})$ The same data as in Panels $\mathrm{D}$ and $\mathrm{E}$, but now the $\left[\mathrm{Ca}^{2+}\right]_{\mathrm{i}}$ has been intensity modulated. As described in Materials and Methods, this effect is obtained by mapping the information in Panel $\mathrm{C}$ to value and the information in Panel $\mathrm{E}$ to hue. 
wound area, which is devoid of cells, the ratio reports a change in $\left[\mathrm{Ca}^{2+}\right]_{\mathrm{i}}$. It is clear from the original pictures that there were no cells in this area; so why is a nonzero ratio produced? Even though the wound area is nearly black, the pixels do have nonzero values. These small intensities are from scattered light and electronic noise. Taking the ratio of small value pixels can lead to large errors, since $1 / 1$ is exactly the same as $255 / 255$. Thus, the original intensity information has been sacrificed for the production of ratiometric $\left[\mathrm{Ca}^{2+}\right]_{\mathrm{i}}$ data.

When a pseudocolor LUT is applied, as in Figure 2E, it is much easier to see which areas are experiencing similar elevations in $\left[\mathrm{Ca}^{2+}\right]_{\mathrm{i}}$. Unfortunately, just like the gray-scale image, information on dye localization is lost, and the wound area still appears to have elevated $\left[\mathrm{Ca}^{2+}\right]_{i}$.

In Figure $2 \mathrm{~F}$, the $\left[\mathrm{Ca}^{2+}\right]_{\mathrm{i}}$ information in Panel $\mathrm{E}$ and the intensity information in Panel $\mathrm{C}$ have been combined using the method outlined in Materials and Methods. The $\left[\mathrm{Ca}^{2+}\right]_{i}$ data are still represented by the hue of the colors. The ability to easily distinguish between small changes and identify equivalent regions is retained by the hue. Furthermore, the re-introduction of the intensity data that were initially acquired, allows the viewer to see the outline of cells and even some cellular structure like the nucleus. Also the artifacts that were introduced by taking the ratio have been masked. This is most dramatic in the wound area, which is now black, and correctly reflects that no useful $\left[\mathrm{Ca}^{2+}\right]_{\mathrm{i}}$ data were collected there.

\section{DISCUSSION}

The use of pseudocolor to display $\left[\mathrm{Ca}^{2+}\right]_{\mathrm{i}}$ is immensely popular. However, most pictures display only the $\left[\mathrm{Ca}^{2+}\right]_{i}$ data and throw out the intensity information that was collected. This can lead to the inclusion of errors in the resulting ratio picture. This is most often accounted for by using a threshold value when obtaining ratios. A threshold value is picked by the experimenter, and if a pixels intensity from either the 340 or $385 \mathrm{~nm}$ image drops below that value, the ratio is set to zero. This has several drawbacks. First, the threshold value must be picked for each individual run. This is an arbitrary process and introduces a new opportunity for experimental bias. Second, this technique clips out the data that failed the researchers arbitrary criteria, but fails to show the relative strength or weakness of the original signal of the remaining pixels. By using intensity modulation, it is possible to remove the artifacts and provide a weighted visual measure of the original signal-to-noise ratio of the data that were acquired. Furthermore, the final image is visually rich, containing both $\left[\mathrm{Ca}^{2+}\right]_{\mathrm{i}}$ and morphological data as shown by dye localization.

When implementing intensity modulation, the researcher has a choice between performing this with hardware or software. This had been done previously with hardware $(4,7)$. We feel that a software solution offers several tangible benefits to the researcher. ( $i)$ The first benefit is freedom-the method is independent of operating system, camera, digitizing board or acquisition software. Data from dramatically different microscopes can be viewed in exactly the same way. (ii) The second benefit is in integration-a software solution can be integrated into an existing system without disturbing the already 
established acquisition phase. Or, intensity modulation could be added as an integral part of the acquisition phase. (iii) The third major benefit is price-software is much cheaper than hardware and is far simpler to upgrade and maintain.

The success of this method is reliant on the choice of LUT. It is absolutely critical that the colors used for the LUT do not vary in value. If the colors were to vary in value, then when the isobestic was used to replace the value component of the image, the colors would no longer be calibrated to $\left[\mathrm{Ca}^{2+}\right]_{\mathrm{i}}$. It would however be possible to use colors that were not fully saturated. We have chosen $100 \%$ saturation because it provides the maximum separation of distinct colors and is visually well matched to the $\left[\mathrm{Ca}^{2+}\right]_{\mathrm{i}}$ data.

This method produces images that are easy to reproduce on the printed page. Most often color images are printed using Cyan Yellow Magenta Black (CMYK) process inks. Unfortunately, the gamut of possible colors in the RGB space of a computer monitor is greater than that of CMYK (6). Deep, brilliant, fully intense and fully saturated blues fall outside the CMYK gamut and are just not possible in CMYK space. When trying to print this kind of blue in CMYK, the color that comes out is usually a gray blue and does not fit with the other fully saturated colors. This is why we recommend leaving blue out of your LUT; start at cyan and move around the cone (Figure 1B) stopping at magenta. Red and green also suffer in CMYK space but not as dramatically as blue. Reds and greens usually come out darker than expected but are still close enough to full saturation so as not to look out of place.

This method also introduces a new possibility for quantitation of $\left[\mathrm{Ca}^{2+}\right]_{i}$. Currently, when measurements are done on ratio pictures, a region of interest (ROI) is picked by the user, and the mean change in pixel intensity is recorded in this area over time. All the pixels in the ROI are counted equally, regardless of the quality of the acquired data. By incorporating features of this technique while recording the mean change over time, a more accurate quantitation of $\left[\mathrm{Ca}^{2+}\right]_{i}$ could be obtained.

The hue of the color corresponds directly to the level of $\left[\mathrm{Ca}^{2+}\right]_{\mathrm{i}}$ in the cell. The intensity of the color is analogous to the certainty of the measurement. If a cell has very little dye, the signal will be weak, and errors in recording the intensity will increase. The error in the ratio will increase even more since the emitted intensity at 385-nm excitation gets dimmer with increased $\left[\mathrm{Ca}^{2+}\right]_{\mathrm{i}}$. Any $\left[\mathrm{Ca}^{2+}\right]_{\mathrm{i}}$ values obtained are likely to approach the lower limit of the detector and thus give erroneously high numbers. These errors could all be avoided by weighting the ratio measurements with the intensity measurements. Thus an area with a high intensity will be counted more heavily than an area with low intensity. A formula such as Equation 1 can be used.

$$
\bar{x}_{w}=\frac{w_{1} x_{1}+w_{2} x_{2}+\cdots w_{n} x_{n}}{w_{1}+w_{2}+\cdots w_{n}}
$$

Where $\bar{x}_{w}$ is the weighted average, $w$ is the intensity and $x$ is the calculated $\left[\mathrm{Ca}^{2+}\right]_{\mathrm{i}}$. This produces a weighting of the data, which is the same at the visual weighting achieved by intensity modulation. With the increased availability of cheap and powerful computers, such calculations are relatively rapid, and the increase in accuracy of the measurement is well worth the cost of increased computational time.

\section{ACKNOWLEDGMENTS}

We would like to thank Barry Schaudt from the Minnesota Super Computer Institute for his effort and assistance in creating Figure 1 and Gerry Sedgewick for his assistance with Photoshop. We would also like to thank Greg Beilman and Kristine Groehler for isolating and characterizing rat alveolar Type II cells. This work was supported by National Science Foundation Grant No. MCB-9596838 to P.J.S.

\section{REFERENCES}

1.Foley, J. and A. Van Dam. 1982. Chapter 17. In Fundamentals of Interactive Computer Graphics. Addison Wesley, Reading, MA.

2.Garcia-Sancho, J., M. Alonso and A. Sanchez. 1989. Receptor-operated calcium channels in human platelets. Biochem. Soc. Trans. 17:980-982.

3.Grynkiewicz, G., M. Poenie and R. Tsien. 1985. A new generation of $\mathrm{Ca}^{2+}$ indicators with greatly improved fluorescence properties. J. Biol. Chem. 260:3440-3450.

4.Gustafson, M. and K.E. Magnusson. 1992. A novel principle for quantitation of fast intra-cellular calcium changes using Fura- 2 and a modified image processing system-application in studies of neutrophil motility and phagocytosis. Cell Calcium 13:473-486.

5.Hinman, L., G. Beilman, K. Groehler and P. Sammak. 1997. Woundinduced calcium waves in alveolar type II cells. Am. J. Physiol. 273:L1242-1248.

6.Russ, J.C. 1995. Chapter 1. In The Image Processing Handbook. CRC Press, Boca Raton.

7.Tsien, R. and A. Harootunian. 1990. Practical design criteria for a dynamic ratio imaging system. Cell Calcium 11:93-109.

Received 23 December 1997; accepted 31 March 1998.

Address correspondence to:

Dr. Paul J. Sammak

Department of Pharmacology

University of Minnesota

3-249 Millard Hall

435 Delaware St. S.E.

Minneapolis, MN 55455, USA

Internet: sammak@maroon.tc.umn.edu 